Fermi gases with imaginary mass imbalance and the sign problem in Monte-Carlo calculations

This content has been downloaded from IOPscience. Please scroll down to see the full text.

2014 J. Phys. G: Nucl. Part. Phys. 41055110

(http://iopscience.iop.org/0954-3899/41/5/055110)

View the table of contents for this issue, or go to the journal homepage for more

Download details:

IP Address: 140.112.101.4

This content was downloaded on 12/11/2014 at 03:44

Please note that terms and conditions apply. 


\title{
Fermi gases with imaginary mass imbalance and the sign problem in Monte-Carlo calculations
}

\author{
Dietrich Roscher ${ }^{1}$, Jens Braun ${ }^{1,2}$, Jiunn-Wei Chen ${ }^{3}$ \\ and Joaquín E Drut ${ }^{4}$ \\ ${ }^{1}$ Institut für Kernphysik (Theoriezentrum), Technische Universität Darmstadt, \\ D-64289 Darmstadt, Germany \\ ${ }^{2}$ ExtreMe Matter Institute EMMI, GSI, Planckstraße 1, D-64291 Darmstadt, Germany \\ ${ }^{3}$ Department of Physics, National Center for Theoretical Sciences and Leung Center \\ for Cosmology and Particle Astrophysics, National Taiwan University, Taipei 10617, \\ Taiwan \\ ${ }^{4}$ Department of Physics and Astronomy, University of North Carolina, Chapel Hill, \\ NC 27599, USA
}

E-mail: drosch@theorie.ikp.physik.tu-darmstadt.de

Received 17 December 2013, revised 10 February 2014

Accepted for publication 11 February 2014

Published 19 March 2014

\begin{abstract}
Fermi gases in strongly coupled regimes are inherently challenging for manybody methods. Although progress has been made analytically, quantitative results require $a b$ initio numerical approaches, such as Monte-Carlo (MC) calculations. However, mass-imbalanced and spin-imbalanced gases are not accessible to MC calculations due to the infamous sign problem. For finite spin imbalance, the problem can be circumvented using imaginary polarizations and analytic continuation, and large parts of the phase diagram then become accessible. We propose to apply this strategy to the mass-imbalanced case, which opens up the possibility to study the associated phase diagram with MC calculations. We perform a first mean-field analysis which suggests that zero-temperature studies, as well as detecting a potential (tri)critical point, are feasible.
\end{abstract}

Keywords: many-body theory, mass-imbalance, Fermi gases, Monte-Carlo simulations

(Some figures may appear in colour only in the online journal) 


\section{Introduction}

Ultracold Fermi gases have attracted considerable interest in the past 15 years. The sustained level of activity in this field can be traced back to the fact that quantum many-body phenomena $[1,2]$, ranging from Bose-Einstein condensation to Bardeen-Cooper-Schrieffer superfluidity, have become accessible to experiments in a progressively cleaner and more versatile way. Experimental control parameters include the (local) density $n$ and the s-wave scattering length $a_{\mathrm{s}}$. The latter can be tuned by an external magnetic field in the presence of a Feshbach resonance. For a sufficiently dilute Fermi gas, the range of the interaction effectively represents the smallest length scale in this many-body problem and therefore the dynamics is completely controlled by the dimensionless parameter $k_{\mathrm{F}} a_{\mathrm{s}}$, where the Fermi momentum $k_{\mathrm{F}}$ is determined by the density $n$.

In the limit of large s-wave scattering length (unitary regime) the only scale left is the density $n$. For these systems, a small expansion parameter has not yet been identified and most likely does not exist. Therefore, the use of non-perturbative methods is unavoidable, which makes this limit a major challenge for theoretical approaches [3]. On the other hand, experimental studies have achieved high precision in some cases [4]. Apart from their phenomenological relevance, these experiments can be used to benchmark theoretical methods.

In this work, we restrict ourselves to the case of mass-imbalanced Fermi gases in the unitary regime, i.e. we focus on Fermi gases consisting of two species with unequal masses interacting resonantly with each other. To study such a Fermi gas, great efforts have been made in recent years, both on the experimental [5] and the theoretical side (see, e.g., [6] for a diffusion Monte-Carlo (MC) calculation and, e.g., [7] for a more general review). Nevertheless, mass-imbalanced Fermi gases remain out of reach for $a b$ initio MC calculations due to the infamous sign problem (see, e. g., [8]). On the other hand, the mass-balanced unitary Fermi gas is free of these complications $[9,10]$ and shows good agreement with experimental data [11]. The situation resembles that of polarized Fermi gases, which are also affected by the sign problem in MC approaches. For the latter, however, an approach to avoid the sign problem was put forward in [12]. In analogy to lattice studies of the QCD phase diagram [13], this strategy employs an analytic continuation from real to imaginary polarization which opens up the possibility to study large parts of the phase diagram, potentially including the (tri)critical point.

Inspired by the studies mentioned above, we show here that analytic continuation can be used to study mass-imbalanced Fermi gases with lattice MC calculations, without a sign problem. Moreover, in contrast to the polarized case, we show that the zero-temperature limit is accessible. This opens up the possibility for a detailed comparison of experimental data and results from $a b$ initio lattice MC calculations, which will enhance our understanding of pairing in strongly interacting Fermi gases.

We apply a (standard) mean-field approach to a unitary mass-imbalanced Fermi gas in three (spatial) dimensions, as also discussed elsewhere (see, e.g., [7, 14, 15]). In contrast to earlier works, we employ real- and imaginary mass imbalances, and discuss how the phase diagram for the imaginary case can be used to determine the physical case. We do not expect our mean-field study to yield the accurate values for physical observables. Nevertheless, it can be viewed as the lowest-order approximation and, as is the case for the full evaluation of the associated path integral, it only depends on a single input parameter (e.g. $k_{\mathrm{F}}$ ). Therefore, our results do not suffer from a parameter ambiguity, but only from uncertainties associated with the mean-field approximation, (see, e.g., [16] for a more general discussion of this important issue). We thus expect our mean-field analysis to be suitable to demonstrate our approach, 
allowing us to gain essential insights into the analytic structure of the theory, which can then be used to guide MC calculations.

\section{Formalism}

We proceed by writing the partition function as

$$
\mathcal{Z}\left(T, m_{\uparrow}, m_{\downarrow}, \bar{\mu}, h\right)=\operatorname{Tr}\left[\mathrm{e}^{-\beta\left(\hat{H}-\bar{\mu}\left(\hat{N}_{\uparrow}+\hat{N}_{\downarrow}\right)-h\left(\hat{N}_{\uparrow}-\hat{N}_{\downarrow}\right)\right)}\right],
$$

where $\beta=1 / T$ is the inverse temperature. The Hamiltonian $\hat{H}$ describes a theory with two fermion species, $\uparrow$ and $\downarrow$, with a zero-range interaction:

$$
\hat{H}=\int \mathrm{d}^{3} x\left(-\sum_{\sigma=\uparrow, \downarrow} \hat{\psi}_{\sigma}^{\dagger}(\mathbf{x}) \frac{\vec{\nabla}^{2}}{2 m_{\sigma}} \hat{\psi}_{\sigma}(\mathbf{x})+\bar{g} \hat{\rho}_{\uparrow}(\mathbf{x}) \hat{\rho}_{\downarrow}(\mathbf{x})\right) .
$$

The operators $\hat{\rho}_{\uparrow, \downarrow}$ are the particle-density operators, and $\hat{N}_{\uparrow, \downarrow}$ are the corresponding particlenumber operators. The masses of the two species are given by $m_{\uparrow}$ and $m_{\downarrow}$, respectively. We have also introduced the average chemical potential $\bar{\mu}=\left(\mu_{\uparrow}+\mu_{\downarrow}\right) / 2$ and the asymmetry parameter $h=\left(\mu_{\uparrow}-\mu_{\downarrow}\right) / 2$. From now on, however, we consider the case $h=0$ and allow only for a finite mass imbalance. To this end, it is convenient to introduce quantities that allow us to measure the mass imbalance in simple terms:

$$
m_{+}=\frac{4 m_{\uparrow} m_{\downarrow}}{m_{\uparrow}+m_{\downarrow}}, \quad m_{-}=\frac{4 m_{\uparrow} m_{\downarrow}}{m_{\downarrow}-m_{\uparrow}}, \quad \bar{m}=\frac{m_{+}}{m_{-}} .
$$

The dimensionless imbalance parameter $\bar{m}=\left(m_{\downarrow}-m_{\uparrow}\right) /\left(m_{\downarrow}+m_{\uparrow}\right)$ measures the relative strength of the mass imbalance. Note that $0 \leqslant|\bar{m}|<1$.

MC calculations for unitary fermions with $h=0$ and $\bar{m}=0$ can be performed without a sign problem (see e.g. [8-10]). For the case $h>0$ and $\bar{m}=0$, it was pointed out in [12] that the sign problem can be circumvented using an imaginary-valued asymmetry parameter $h$. This corresponds to a theory with complex-valued $\mu$. The results from such an MC study can then be analytically continued to obtain the results for the physically interesting case of a real-valued asymmetry parameter. For the case $h=0$ and $\bar{m}>0$, a similar approach can be used to avoid the appearance of the sign problem in MC calculations. Assuming $m_{-}$is imaginary-valued, it is indeed straightforward to show that the fermion determinants appearing in the probability measure are complex conjugates of one another, provided that the parameter $m_{+}$is still considered to be real-valued and $m_{\uparrow}^{*}=m_{\downarrow}$. For convenience, we define $\bar{m}=\mathrm{i} \bar{m}_{\mathrm{I}}$ with $\bar{m}_{\mathrm{I}} \in \mathbb{R}$. Fermi gases with imaginary mass imbalance can thus be studied using standard $\mathrm{MC}$ techniques. As in the case of imaginary-valued $h$, the actual results of physical interest are to be found by analytically continuing to the real axis, to obtain the original partition function $\mathcal{Z}\left(T, m_{\uparrow}, m_{\downarrow}, \bar{\mu}, h\right)$ with $m_{\uparrow, \downarrow} \in \mathbb{R}$. From the latter we can, in principle, extract all observable equilibrium quantities.

To illustrate our imaginary mass-imbalance approach and to gain deeper insights into the analytic structure of the theory (which is required to guide future MC studies), we employ a mean-field study with complex-valued masses $m_{\uparrow, \downarrow}$, such that $m_{\uparrow}^{*}=m_{\downarrow}$. The real and imaginary parts of these masses can then be tuned to obtain a certain given value for the imbalance parameter $\bar{m}$. To compute the order-parameter potential for U(1) symmetry breaking in the mean-field approximation, we employ the path-integral representation of $\mathcal{Z}$ : 


$$
\begin{aligned}
& \mathcal{Z}=\int \mathcal{D} \psi^{\dagger} \mathcal{D} \psi \mathrm{e}^{-S\left[\psi^{\dagger}, \psi\right]}, \\
& \begin{array}{l}
S\left[\psi^{\dagger}, \psi\right]=\int \mathrm{d} \tau \int \mathrm{d}^{3} x\left\{\psi^{\dagger}\left(\partial_{\tau}-\frac{1}{m_{+}} \vec{\nabla}^{2}-\bar{\mu}\right) \psi\right. \\
\left.\quad-\frac{1}{m_{-}}\left(\psi_{\uparrow}^{*} \vec{\nabla}^{2} \psi_{\uparrow}-\psi_{\downarrow}^{*} \vec{\nabla}^{2} \psi_{\downarrow}\right)+\bar{g}\left(\psi^{\dagger} \psi\right)\left(\psi^{\dagger} \psi\right)\right\},
\end{array}
\end{aligned}
$$

and $\psi^{\mathrm{T}}=\left(\psi_{\uparrow}, \psi_{\downarrow}\right)$ and $\bar{g}$ is the bare coupling. The latter is related to the scattering length $a_{\mathrm{s}}$ by $\bar{g}^{-1}=\Lambda g^{-1}=\left(a_{\mathrm{s}}^{-1}-c_{\text {reg. }} \Lambda\right) / 8 \pi$. Here, $\Lambda$ is the ultraviolet cutoff and the constant $c_{\text {reg. }}>0$ depends on the regularization scheme. We choose units such that $m_{+}=1$ corresponding to $2 m=1$ for $m_{\uparrow}=m_{\downarrow}=m$. This choice also implies that $\bar{m}=1 / m_{-}$.

We proceed by introducing an auxiliary scalar field $\varphi \sim g_{\varphi} \psi_{\uparrow} \psi_{\downarrow}$, where the parameter $g_{\varphi}$ is chosen to reproduce the four-fermion term in the action. The fermion fields then only appear bilinearly in the action and can be integrated out. Assuming a homogeneous ground state, the resulting fermion determinants can be computed straightforwardly, eventually yielding the order-parameter potential:

$\beta U(\varphi)=-2 \beta \bar{\mu}|\varphi|^{2}-\int \frac{\mathrm{d}^{3} q}{(2 \pi)^{3}} \ln \left[\cosh \left(\beta \bar{m} \vec{q}^{2}\right)+\cosh \left(\beta \sqrt{\left(\vec{q}^{2}-\bar{\mu}\right)^{2}+g_{\varphi}^{2}|\varphi|^{2}}\right)\right]$.

For conciseness, we have dropped the standard terms that regularize the potential (see, e.g., $[7,15])$.

The order-parameter potential $U$ and the grand canonical potential $\Omega$ are directly related, $\Omega=V U\left(\varphi_{0}\right)$, where $V$ is the volume of the system and $\varphi_{0}$ denotes the value of $\varphi$ that minimizes the potential. In the ground state, we can identify $g_{\varphi}^{2}\left|\varphi_{0}\right|^{2}$ with the fermion gap $\Delta$. The latter serves as an order parameter for spontaneous U(1) symmetry breaking associated with a superfluid ground state. From $\Omega$ we can extract all thermodynamic observables. As it should be, our results for dimensionless (universal) quantities in the unitary limit, such as the critical temperature $T_{\mathrm{c}}(\bar{m}) / \bar{\mu}$ for the superfluid transition or the ground-state energy $E(\bar{m}) / \bar{\mu}$, are independent of $\bar{\mu}$ and $g_{\varphi}$.

\section{Results}

For our discussion of some of the analytic properties of the theory, it is instructive to briefly compare the mass-imbalanced case with the spin-imbalanced case. For $\bar{m}=0$ and $h>0$, the theory is $2 \pi$-periodic in $\beta h_{\mathrm{I}}$, where $h=\mathrm{i} h_{\mathrm{I}}$ and $h_{\mathrm{I}} \in \mathbb{R}$ (see [12]). Thus, for imaginary spin imbalances the accessible polarizations are bound by $\beta h_{\mathrm{I}}<\pi$. For the mass-imbalanced case, we do not have periodic behavior in $\bar{m}$, as the parameters for mass imbalance and spin imbalance are associated with different operators (cf equation (2)). However, we do find that the zero-temperature limit is accessible in the case of imaginary-valued mass asymmetries.

For our purposes, the analytic continuation from imaginary-valued to real-valued mass asymmetries requires the theory to be analytic in a finite domain around the symmetric point $\bar{m}=0 .{ }^{5}$ For given fixed values of $\varphi, T$, and $\mu$, we find that the order-parameter potential

5 Note that our analysis of the (practical) applicability of an imaginary mass imbalance to studies of mass-imbalanced Fermi gases is based on our mean-field study with a homogeneous background field $\varphi$. In studies beyond the meanfield approximation, where also fluctuations about the background field $\varphi$ are taken into account, the radius of convergence may be different. An estimate of whether the radius of convergence becomes larger or smaller beyond our mean-field approximation is beyond the scope of this work. In any case, we stress that our underlying idea of curing the sign problem in mass-imbalanced lattice MC studies by using an imaginary mass imbalance is not affected by such fluctuation effects. The fermion determinants appearing in the probability measure of a lattice MC calculation are complex conjugates of one another in the case of an imaginary mass imbalance. Fluctuation effects present in these studies do not alter our line of arguments in this respect. 


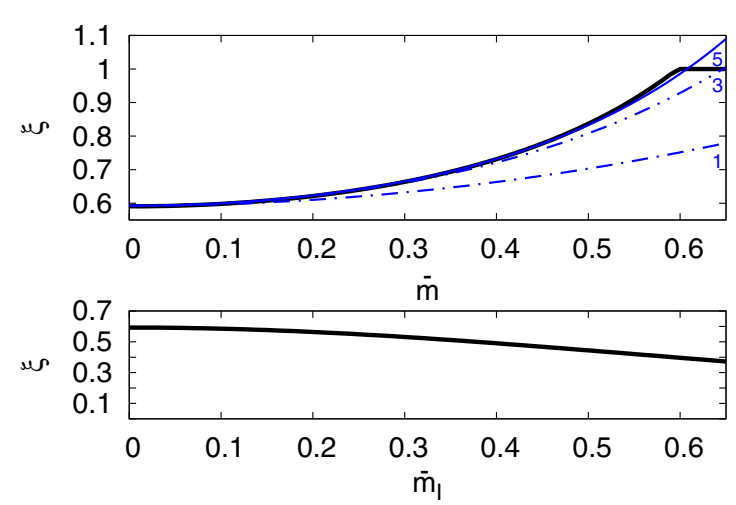

Figure 1. Upper panel: Bertsch parameter as a function of $\bar{m}$. The (blue) thin dasheddotted lines are analytic continuations obtained from a Taylor expansion of the Bertsch parameter in $\bar{m}_{\mathrm{I}}^{2}$ up to order $N_{\max }$ (indicated by the numbers, see text). Lower panel: Bertsch parameter as a function of $\bar{m}_{\mathrm{I}}$.

(3) can indeed be expanded in powers of $\bar{m}$. From the analytic structure of the integrand in equation (3), it follows that a lower bound $r_{\min }$ for the radius of convergence $r_{\bar{m}}$ of such an expansion is given by

$$
r_{\min }\left(|\Phi|^{2}\right)=\sqrt{\frac{\beta^{2}|\Phi|^{2}+\pi^{2}}{\beta^{2}|\Phi|^{2}+\pi^{2}+\beta^{2} \bar{\mu}^{2}}},
$$

where $|\Phi|^{2}=g_{\varphi}^{2}|\varphi|^{2}$. This lower bound remains finite as $\beta \rightarrow \infty$. To compute physical observables $\mathcal{O}$, such as the density or the ground-state energy, we need to evaluate the potential at its (global) minimum $\varphi_{0}$. The radius $r_{\bar{m}, \mathcal{O}}$ for an observable $\mathcal{O}$ is then bounded from below by $r_{\min }\left(\Delta=g_{\varphi}^{2}\left|\varphi_{0}\right|^{2}\right)$, provided that the integrand in equation (3) is holomorphic in $\Delta$ and $\Delta$ is holomorphic in $\bar{m}$. For $\bar{m}<\bar{m}_{\mathrm{c}}(T)$, we expect this to be the case. Here, $\bar{m}_{\mathrm{c}}(T)$ denotes the critical mass imbalance below which the gap is finite for a given temperature $T$. The actual radius of convergence may well be larger than this lower bound since the analytic properties of the potential may be improved by the momentum integration in equation (3). In the superfluid phase, on the other hand, an upper bound for the radius of convergence is given by $\bar{m}_{\mathrm{c}}(T)$, i.e. $r_{\bar{m}, \mathcal{O}}<\bar{m}_{\mathrm{c}}(T)$. For the energy of the system being measured in terms of the so-called Bertsch parameter $\xi$ (see below for its definition), it indeed appears to be the case that $r_{\bar{m}, \xi} \approx \bar{m}_{\mathrm{c}}$ at $T=0$ (see figure 1 and our discussion below). At high-temperatures $T \gg T_{\mathrm{c}}(\bar{m}=0)$, the gap is zero for $\bar{m}_{(\mathrm{I})} \in[0,1]$ and we have $r_{\bar{m}, \mathcal{O}} \leqslant 1$.

For MC calculations, these findings suggest to compute physical observables as function of $\bar{m}_{\mathrm{I}}$, with standard techniques, without suffering from the sign problem. The results of physical interest, i.e. for real-valued $\bar{m}$, can then be obtained by fitting the MC data to a polynomial ansatz in $\bar{m}_{\mathrm{I}}$ and analytically continuing this polynomial to the real axis. Only even powers of $\bar{m}$ can contribute as the partition function is invariant under the transformation $\bar{m} \rightarrow-\bar{m}$.

We now demonstrate the analytic continuation procedure. For illustration purposes, we fit our mean-field results for a given observable $\mathcal{O}$, such as the Bertsch parameter or $T_{\mathrm{c}}$, to the ansatz $\mathcal{O}=\sum_{n=0}^{N_{\max }} C_{\mathcal{O}}^{(n)} \bar{m}_{\mathrm{I}}^{2 n}$, where $C_{\mathcal{O}}^{(n)}$ are constants determined by the fit and $N_{\max }$ represents the truncation order. (In a lattice MC study, for example, the latter is limited by the amount of available data. While the analytic continuation can in principle be performed in a variety of ways, the underlying MC data would still contain useful non-perturbative information.) 


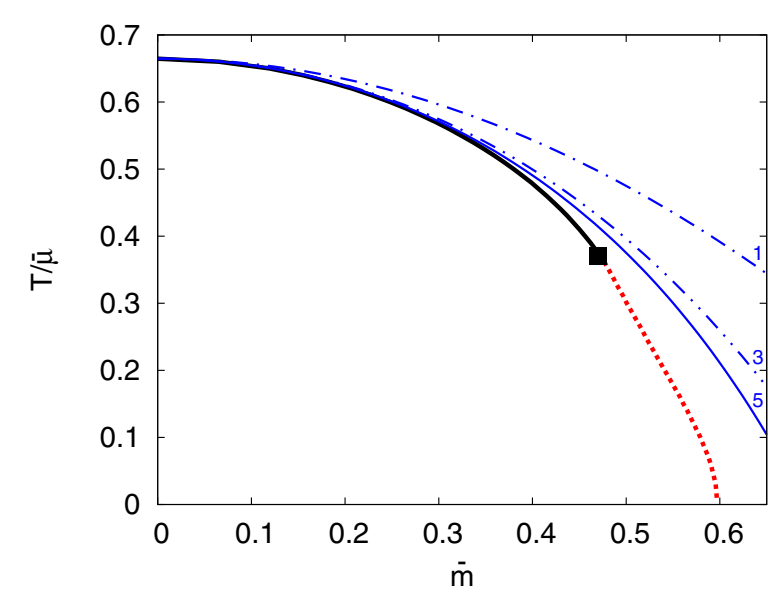

Figure 2. Phase diagram of an ultracold Fermi gas at unitarity in the $(T, \bar{m})$ plane. The solid (black) curve is a line of second-order phase transitions, which ends at a (tri)critical point $\left(\bar{m}_{\mathrm{cp}}, T_{\mathrm{cp}} / \bar{\mu}\right)$ marked by the black square. For $\bar{m}>\bar{m}_{\mathrm{cp}}$, we find a line of firstorder transitions. The (blue) thin dashed-dotted lines are analytic continuations obtained from a Taylor expansion of the phase boundary in $\bar{m}_{\mathrm{I}}^{2}$ up to order $N_{\max }$ (indicated by the numbers, see text).

From a simple analytic continuation of this polynomial, one then obtains the dependence of $\mathcal{O}$ on $\bar{m}$. Within our analytic study, we can easily check the feasibility of such a procedure. In figure 1, we show our results for the Bertsch parameter as a function of $\bar{m}$ as obtained from such a fit procedure. We defined the scale $\epsilon_{\mathrm{F}}$ entering the definition of the Bertsch parameter $\xi=\bar{\mu} / \epsilon_{\mathrm{F}}$ as

$$
\epsilon_{\mathrm{F}}=\left(1-\bar{m}^{2}\right)\left[(1-\bar{m})^{\frac{3}{2}}+(1+\bar{m})^{\frac{3}{2}}\right]^{-\frac{2}{3}}\left(6 \pi^{2} n\right)^{\frac{2}{3}},
$$

where $n=n_{\uparrow}+n_{\downarrow}$ is the total density. With this definition of the Bertsch parameter, we have $\xi=1$ for the free Fermi gas at $T=0$ and $\bar{m} \in[0,1]$. For the interacting gas at $T=\bar{m}=0$, we recover the standard mean-field result $\xi=0.59 \ldots[3,7]$. For the fit presented in figure 1 we have used the results for $\xi$ for 101 equidistant values of $\bar{m}_{\mathrm{I}} \in[0,1]$. For $N_{\max }=5$, we already see good agreement between the analytically continued polynomial and the mean-field result for $\bar{m} \lesssim \bar{m}_{\mathrm{c}}$. A polynomial ansatz is the simplest choice for a fit; more elaborate functions, such as Padé approximants, can also be employed.

With our imaginary mass-imbalance approach, it is also possible to study the thermal properties, as the $T$ dependence of the radius of convergence already implies. The critical temperature $T_{\mathrm{c}}(\bar{m})$ can be computed by studying the gap as a function of $T$ for a given value of $\bar{m}$. Specifically, $T_{\mathrm{c}}(\bar{m})$ is the lowest temperature for which the gap vanishes identically. Since $T_{\mathrm{c}}$ is defined implicitly by the gap, it appears to be impossible to derive the radius of convergence for $T_{\mathrm{c}}(\bar{m})$ analytically. In the following, we shall assume that the radius of convergence for $T_{\mathrm{c}}$ is finite, as suggested by the analytic properties of $U$. We expect the radius of convergence for $T_{\mathrm{c}}(\bar{m})$ to be bounded from above by the radius of convergence of $U$. These statements agree with our numerical results. In figure 2 we show the mean-field phase diagram in the $(T, \bar{m})$ plane. For $\bar{m}<\bar{m}_{\mathrm{cp}}$, the finite-temperature phase transition is found to be of second order, whereas it is of first order for $\bar{m}>\bar{m}_{\mathrm{cp}}$. The (tri)critical point $\left(T_{\mathrm{cp}} / \bar{\mu}, \bar{m}_{\mathrm{cp}}\right) \approx(0.37,0.47)$ is located within the radius of convergence of the order-parameter potential when expanded in powers of $\bar{m}$. This holds true even for $|\Phi|=0$. These observations are intriguing as they 


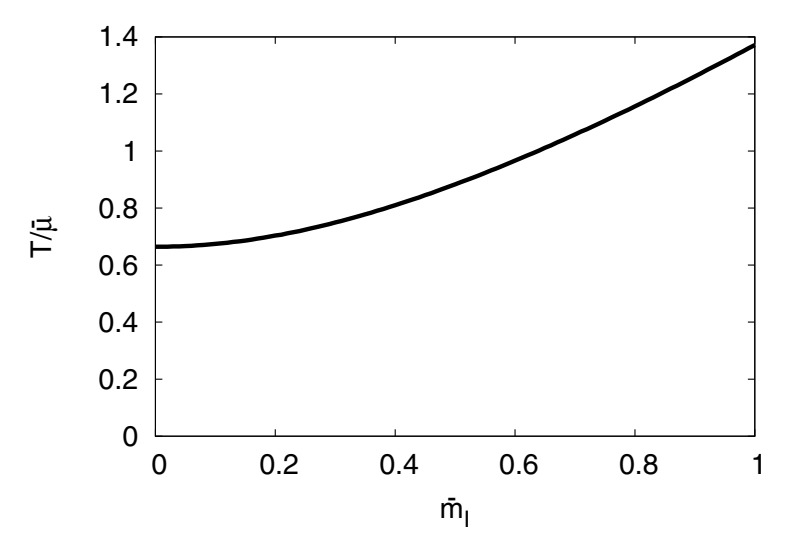

Figure 3. Phase diagram in the $\left(T, \bar{m}_{\mathrm{I}}\right)$ plane. The solid line is a line of second-order phase transitions below which the fermion gap is finite. Note that we do not find a line of first-order phase transitions in this case.

suggest that the (tri)critical point is within the reach of MC calculations with imaginary mass imbalances.

In MC calculations, one would first compute the phase diagram in the $\left(T, \bar{m}_{\mathrm{I}}\right)$ plane with standard techniques without suffering from the sign problem. In figure 3, we show the corresponding phase diagram as obtained from our mean-field approximation. We find that the phase transition line is of second order only and the phase transition temperature is finite for $\bar{m}_{\mathrm{I}} \leqslant 1$. Thus, the (tri)critical point leaves no obvious trace of its existence in this phase diagram. However, the information of the existence of this point is encoded in the shape of $U$ at $\left(\bar{m}_{\mathrm{cp}}, T_{\mathrm{cp}} / \bar{\mu}\right)$. In analogy to the case of imaginary polarization of [12] (see [17] for a relativistic case), we expect the analytic continuation of the phase transition line to give the correct behavior only up to the (tri)critical point. By analytically continuing the (full) orderparameter potential, however, the physical phase diagram in the whole domain of convergence can be recovered, including the line of first-order transitions.

In figure 2 , we show the results for the finite-temperature phase boundary in the $(T, \bar{m})$ plane as obtained from the analytic continuation of the function $T_{\mathrm{c}}\left(\bar{m}_{\mathrm{I}}\right)$ depicted in figure 3 . For the analytic continuation, we have employed polynomials in $\bar{m}_{\mathrm{I}}^{2}$ up to order $N_{\max }$ (see our discussion above). For the fit to our mean-field results, we have used the results for $T_{\mathrm{c}}\left(\bar{m}_{\mathrm{I}}\right) / \bar{\mu}$ for 101 equidistant values of $\bar{m}_{\mathrm{I}} \in[0,1]$. For $N_{\max }=5$ and $\bar{m} \lesssim 0.4$, we already observe good agreement between the analytically continued polynomial and the exact (mean-field) result, see figure 2 .

\section{Summary}

In summary, we have studied the thermodynamics of mass-imbalanced Fermi gases using complex-valued fermion masses. This approach avoids the sign problem in MC calculations, which opens the possibility for ab initio MC studies of mass-imbalanced Fermi gases, in particular at unitarity. The (tri)critical point is in principle within reach in this framework. The zero-temperature limit is directly accessible as well, at least up to a certain value of $\bar{m}$ implicitly determined by the analytic properties of the (full) theory. It should be emphasized again that our analysis of the applicability of our imaginary mass-imbalance approach relies on a mean-field approximation. A lattice MC study based on this approach is deferred to future 
work. In any case, as studies of mass-imbalanced Fermi gases are highly challenging on both the experimental and theoretical side, this work is an important development alongside [12]. Based on this complex-masses approach, future MC studies may indeed allow us to gain deep insights into the collective dynamics underlying strongly interacting Fermi gases which is of great importance for a large variety of systems, ranging from superconducting materials in condensed-matter physics to the nuclear many-body problem.

\section{Acknowledgments}

JB and DR acknowledge support by the DFG under grant BR 4005/2-1 and by HIC for FAIR within the LOEWE program of the State of Hesse. J-WC is supported by the NSC (99-2112M-002-010-MY3) of ROC and CASTS \& CTS of NTU. This work was supported in part by the US National Science Foundation under grant no PHY1306520.

\section{References}

[1] Bloch I, Dalibard J and Zwerger W 2008 Rev. Mod. Phys. 80885 Giorgini S, Pitaevskii L P and Stringari S 2008 Rev. Mod. Phys. 801215

[2] Inguscio M, Ketterle W and Salomon C (ed) 2008 Ultracold Fermi Gases (Proceedings of the International School of Physics 'Enrico Fermi', Varenna, June 20-30 course 164) (Amsterdam: IOS Press)

[3] Zwerger W (ed) 2011 BCS-BEC Crossover and the Unitary Fermi Gas (Berlin: Springer)

[4] Nascimbène S et al 2011 Phys. Rev. Lett. 106215303

Van Houcke K et al 2012 Nature Phys. 8366

Hoinka S et al 2013 Phys. Rev. Lett. 110055305

[5] Wille E et al 2008 Phys. Rev. Lett. 100053201

Voigt A-C et al 2009 Phys. Rev. Lett. 102020405

Trenkwalder A et al 2011 Phys. Rev. Lett. 106115304

Ridinger A et al 2011 Eur. Phys. J. D 65223

Kohstall C et al 2012 Nature $\mathbf{4 8 5} 615$

[6] Gezerlis A et al 2009 Phys. Rev. Lett. 103060403

[7] Gubbels K B and Stoof H T C 2012 arXiv:1205.0568

[8] Drut J E and Nicholson A N 2013 J. Phys. G: Nucl. Part. Phys. 40043101

[9] Chen J-W and Kaplan D B 2004 Phys. Rev. Lett. 92257002

[10] Bulgac A, Drut J E and Magierski P 2006 Phys. Rev. Lett. 96090404

Burovski E et al 2006 Phys. Rev. Lett. 96160402

Lee D and Schafer T 2006 Phys. Rev. C 73015201

Lee D and Schafer T 2006 Phys. Rev. C 73015202

[11] Drut J E et al 2012 Phys. Rev. A 85051601

[12] Braun J, Chen J-W, Deng J, Drut J E, Friman B, Ma C-T and Tsai Y-D 2013 Phys. Rev. Lett. 110130404

[13] de Forcrand P and Philipsen O 2002 Nucl. Phys. B 642290

D'Elia M and Lombardo M-P 2003 Phys. Rev. D 67014505

de Forcrand P and Philipsen O 2007 J. High Energy Phys. JHEP01(2007)077

[14] Baarsma J E, Gubbels K B and Stoof H T C 2010 Phys. Rev. A 82013624

[15] Chevy F and Mora C 2010 Rep. Prog. Phys. 73112401

[16] Diehl S et al 2010 Ann. Phys. 522615

Braun J 2012 J. Phys. G: Nucl. Part. Phys. 39033001

[17] Karbstein F and Thies M 2007 Phys. Rev. D 75025003 